\title{
Remote ischaemic preconditioning-no effect on clinical outcomes after CABG surgery
}

Remote ischaemic preconditioning (RIPC) has been shown to reduce the extent of myocardial injury in patients undergoing cardiac surgery in small, proof-of-concept trials. In contrast to these findings, investigators of two largescale trials published in The New England Journal of Medicine have now shown no benefits of applying RIPC before CABG surgery.

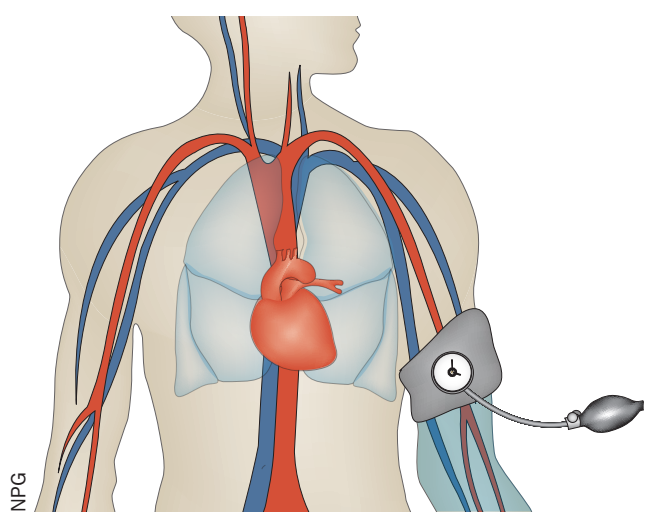

Cardiac surgery carries the risk of end-organ ischaemic and reperfusion injury. "Higher risk patients [are] undergoing $\mathrm{CABG}$ surgery so new treatments [are] required to protect the heart during surgery," explains Derek Hausenloy, one of the investigators. By inflating and deflating a blood-pressure cuff, RIPC delivers short applications of ischaemia and reperfusion to remote organs or tissues. This process is believed to induce adaptive responses that have protective effects on vital organs. "This protective strategy might be a promising key for most of our problems in cardiac surgery," says Patrick Meybohm. The researchers enrolled 1,612 and 1,403 patients undergoing CABG surgery in the two prospective, randomized, sham-controlled trials (ERICCA and RIPHeart). The combined primary end points included, among others, death, myocardial infarction (MI), and stroke. However, the use of RIPC did not improve clinical outcomes in either trial.
Hausenloy now plans to concentrate future research efforts on RIPC in patients with greater myocardial injury, such as ST-segment elevation MI, whereas Meybohm advises focusing on different RIPC protocols or evaluating potential confounders, such as the choice of anaesthesia or co-medications. On the other hand, in an accompanying editorial, Michael Zaugg and Eliana Lucchinetti highlight that although no significant differences in adverse events were observed between the groups, the number of deaths from cardiovascular causes was higher in the RIPC group in one of the trials and, therefore, believe that the safety of RIPC should first be studied.

\section{Clarissa Oeser}

Original articles Hausenloy, D. J. et al. Remote ischemic preconditioning and outcomes of cardiac surgery. N. Engl. J. Med. 373, 1408-1417 (2015) | Meybohm, P. et al. A multicenter trial of remote ischemic preconditioning for heart surgery. N. Engl. J. Med. 373, 1397-1407 (2015) 